\title{
Addressing adenomyosis: Implications beyond we actually know
}

\author{
Swati $^{1 *}$, Ganesh Kumar ${ }^{2}$, Purnima Pachori ${ }^{1}$
}

${ }^{1}$ Department of Obstetrics and Gynecology, JLN Medical College, Ajmer, Rajasthan, India
${ }^{2}$ Department of Pathology, Patliputra Medical College and Hospital, Dhanbad, Jharkhand, India

Received: 17 June 2019

Accepted: 31 July 2019

*Correspondence:

Dr. Swati,

E-mail: somikuki89@gmail.com

Copyright: (c) the author(s), publisher and licensee Medip Academy. This is an open-access article distributed under the terms of the Creative Commons Attribution Non-Commercial License, which permits unrestricted non-commercial use, distribution, and reproduction in any medium, provided the original work is properly cited.

\begin{abstract}
Background: Hysterectomy is one of the commonest gynecological surgeries being performed in India for various pelvic pathologies like fibroid uterus, endometrial hyperplasia, dysfunctional uterine bleeding, etc. But pre-operative diagnosis of adenomyosis and making it an indication for hysterectomy is not as common as pathologists find it in histo-pathology of hysterectomized specimens. The aim of the study was to study the frequency of adenomyosis in comparison to leiomyoma as a uterine pathology in hysterectomized specimens and correlate them clinically.

Methods: A retrospective comparative study was carried out on 1646 hysterectomy specimens, during January 2014 to December 2016, which showed either adenomyosis or leiomyoma or both. Clinical records of these cases were retrieved and histo-pathology was correlated to clinical presentations and pre-operative ultrasonography.

Results: Of the 1646 specimens taken for comparision between adenomyosis and leiomyoma, 49\% showed only adenomyosis, $37 \%$ only leiomyoma and $14 \%$ had dual pathology showing findings of both. The peri-menopausal age group (45-54 years) accounted for the maximum number of patients undergoing hysterectomy (37.12\%). But adenomyosis was found maximum in $35-44$ years age group (38.04\%). The clinical presentations for these two pathologies were similar and maximum patients presented with abnormal uterine bleeding and pelvic pain. Ultrasonography was able to diagnose only $32 \%$ cases of adenomyosis pre-operatively whereas this figure was $87 \%$ for fibroids.

Conclusions: Adenomyosis and leiomyoma both account for the most frequent findings in hysterectomy specimens. Fibroids are easily diagnosed pre-operatively, but adenomyosis needs to be diagnosed pre-operatively by high index of clinical suspicion and imaging techniques. Adenomyosis is not just a disease of middle age, it needs to be addressed for infertility, recurrent pregnancy loss (RPL), bad obstetric history, IVF failures and adherent placenta as well.
\end{abstract}

Keywords: Adenomyosis, AUB, Dysmenorrheal, Fibroid, Hysterectomy, Leiomyoma

\section{INTRODUCTION}

Adenomyosis was first described by Rokitansky in 1860 as "cystosarcoma adenoides uterinum" and was later defined by Von Recklinghausen in 1896. It is a common condition that predominantly affects women in the late reproductive years. Adenomyosis has been noted to specimens depending on the definition of the entity. ${ }^{1}$
Adenomyosis still remains an inaccessible disease for pre-operative diagnosis. Its diagnosis is frequently accomplished retrospectively after hysterectomy. However with use of high resolution imaging techniques a pre-operative diagnosis is possible. On the contrary, leiomyomas are easily diagnosed pre-operatively by clinical examination and simple ultrasonography. Both, adenomyosis and fibroids are responsible for similar 
clinical presentations such as abnormal uterine bleeding, dysmenorrhea, pressure symptoms and infertility.

Despite several advancements in the non-surgical management of various gynecological disorders the rate of hysterectomy is quite high in India for definite management of various uterine pathologies. Today also hysterectomy remains gold standard in the era of minimal access surgery. The various indications for hysterectomy are fibroid, adenomyosis, dysfunctional uterine bleeding (DUB), cervical intra-epithelial neoplasia (CIN), endometrial hyperplasia, chronic pelvic pain (CPP) and post-menopausal bleeding. In past time, fibroid uterus (leading to AUB, pelvic pain and pressure symptoms) has remained the commonest indication for performing hysterectomy. CIN, CPP and post-menopausal bleeding are less frequent causes contributing to the overall rate of hysterectomy. In a last couple of decades, adenomyosis is emerging as a frequent finding post-operatively in the hysterectomy specimens on histo-pathological reporting. Leiomyoma and adenomyosis are seemingly quite frequent causes of AUB and other symptoms for which hysterectomy is being performed.

So, the aim of the retrospective study was to correlate adenomyosis and leiomyoma clinically in terms of prevalence in the region, their clinical presentations and correlation to pre-operative imaging techniques.

\section{METHODS}

A retrospective descriptive study was carried out in the department of obstetrics and gynecology and the department of pathology in JLN Medical College, Ajmer for a period of three years i.e. July 2013 to June 2016. During these three years 1844 hysterectomies were performed in the hospital. Out of these, 1646 hysterectomy specimens were included in this study. Inclusion criteria were those specimens only showing either adenomyosis or leiomyoma or both on histopathology. 198 specimens were excluded from the study in which hysterectomy was performed due to other indications like malignancy, pre-malignant lesions, etc. and the specimens which showed findings other than leiomyoma and adenomyosis were excluded too.

The clinical records of these patients were retrieved and patients' age, parity, clinical presentation, sonography findings and clinical indication for hysterectomy was observed. MRI indicated for some other reasons in a very small number of patients was able to detect these two pathologies pre-operatively. But MRI was not specifically indicated in the majority of patients owing to its cost, so the study was unable to correlate the role of MRI in diagnosis of these two pathologies. Any past history of infertility or poor pregnancy outcomes was also looked for.

The histo-pathological reporting of each specimen was observed in terms of gross and microscopic findings. The microscopic sections were fixed in paraffin and stained by routine Hematoxylin and Eosin stain. The criteria used for the diagnosis of adenomyosis were as follows:

- Grossly - pin-point or small cystic areas of hemorrhage seen within myometrium.

- Microscopically - presence of endometrial glands and stroma in the myometrium more than one low power field away from endo-myometrial junction.

\section{RESULTS}

Out of the total 1844 hysterectomies performed during the study period, $807+230=1037$ specimen showed adenomyosis (807 - adenomyosis; 230 - both adenomyosis and fibroid), so its prevalence was $56.24 \%$ among all hysterectomy patients during the study period. Fibroids showed a prevalence of $45.50 \%$ $(609+230=839)(609$ - fibroid; 230 - both fibroid and adenomyosis). A total of 1646 cases were included in the study. $49 \%$ of these were reported as adenomyosis, whereas $37 \%$ had leiomyoma and $14 \%$ had findings of both adenomyosis and leiomyoma (Figure 1).

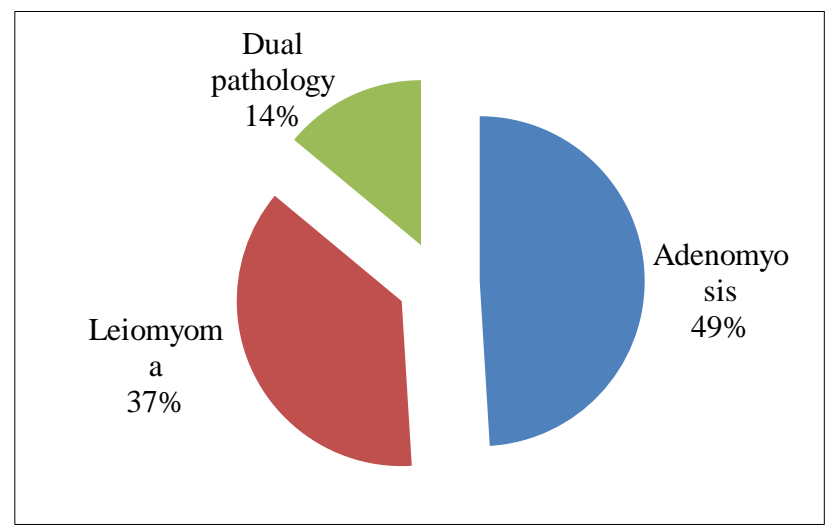

Figure 1: Percentage of each histopathological lesions.

Age of the patients ranged from 28 years to 73 years. The youngest age group (25 - 34 years) undergoing hysterectomy in this retrospective study had completed their child-bearing function in $98.5 \%$ cases. In the remaining $1.5 \%$ cases various reasons for hysterectomy of nulliparous/ primipara patients were multiple fibroid uterus which recurred even after medical management, or non-affordability of costlier drugs by patients, illiteracy and lack of further follow-up with conservative and medical management and most importantly persistence of patients' complaints and hence their will to get rid of their uteri. In this youngest age group, pre-operative indications made for hysterectomy were fibroid uterus with AUB and/or pelvic pain in $53.58 \%$ patients, pelvic inflammatory disease (PID) with/without AUB in $27.30 \%$ cases and adenomyosis in remaining $19.12 \%$ patients. To surprise post-operative histological diagnosis of fibroid was found in $36.86 \%$, adenomyosis in $55.63 \%$ and dual pathology in $7.51 \%$ patients of this age group patients. 
Figure 2 depicts the age distribution of patients as per the type of histo-pathology being studied. The largest group $(n=611)$ was of peri-menopausal age $(45-54$ years $)$ contributing $37.12 \%$ of total cases in the study. This age group had maximum numbers of patients with leiomyoma and dual pathology as well. But adenomyosis was seen at the most in 35 - 44 years age group (38.04\%). The signs and symptoms of both the pathologies were similar in the study subjects and maximum patients presented with more than one symptom (Figure 3 ).

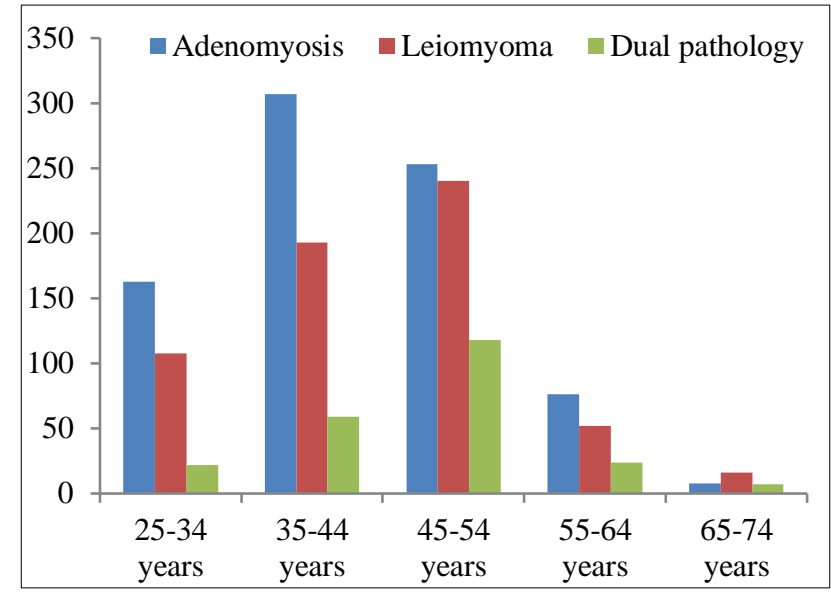

Figure 2: Age distribution of study subjects.

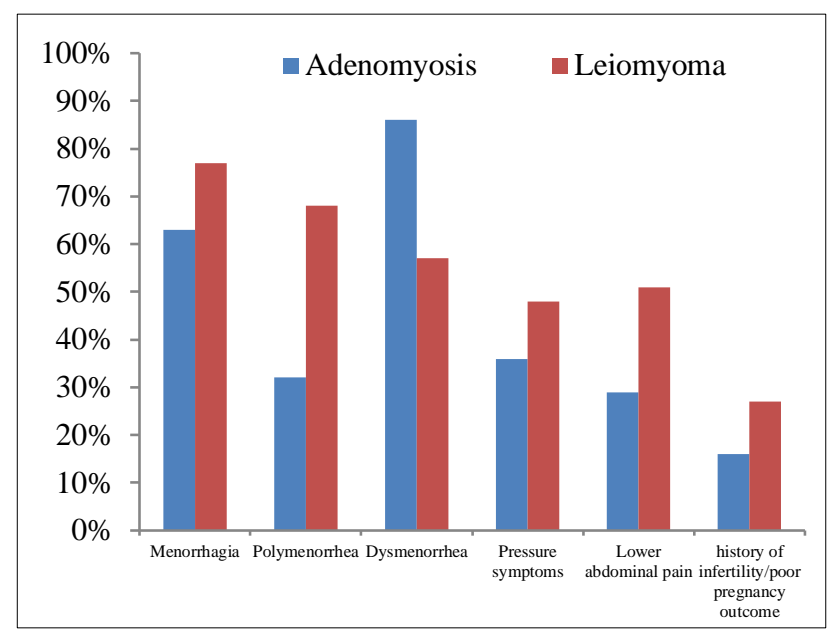

Figure 3: Pattern of clinical presentation by study subjects.

Heavy menstrual bleeding and pelvic pain were the most common complaints with which patients presented in both the pathologies. Of the 839 cases of fibroid uterus, 740 cases were provisionally diagnosed pre-operatively on clinical examination $(88.20 \%)$. Of the 1037 cases of adenomyosis documented histologically, bimanual pelvic examination of 923 cases $(89 \%)$ showed bulky uterus and only 270 cases $(26.04 \%)$ had tenderness also along with bulky uterus. But concomitant complaints of white discharge per vaginum misguided the clinical indication for hysterectomy as PID with AUB in a significant number of cases i.e. $77.91 \%$ (808 cases of adenomyosis).
In $48.02 \%$ cases, adenomyosis was kept as differential diagnoses, which actually were found so on histopathology. Only one fourth fraction of these cases were given non-surgical treatment in form of levonorgestrel-releasing intra-uterine devices and monthly injections of gonadotrophin releasing hormone analogues for three to six months but due to partial relief of symptoms, they opted for hysterectomy. Remaining three-fourth cases could not afford these treatment options due to high cost and in only $32 \%$ of the subjects who had adenomyosis, a pre-operative provisional diagnosis was made on the basis of ultrasonography. But this figure was $87 \%$ in case of leiomyoma (not only in making provisional diagnosis, but also in defining the site and size of fibroids). Most importantly out of remaining $68 \%$ cases in which pre-operative ultrasonography had missed adenomyosis, 52\% cases were diagnosed as PID owing to presence of bulky uterus and free fluid in pouch of Douglas and $48 \%$ were given normal findings in ultrasound scan. Obstetric histories of all the 1037 cases of adenomyosis were reviewed, of which 446 cases (43\%) had past history of either one or more of these: primary or secondary infertility, RPL, preterm deliveries (due to preterm labour or PPROM), adherent placenta and post-partum hemorrhage.

\section{DISCUSSION}

Menorrhagia or hypermenorrhea is defined as abnormally long or heavy menses lasting $>7$ days or involving blood loss $>80 \mathrm{~mL}$. Metrorrhagia means menses occurring at irregular intervals. Polymenorrhea is referred to frequent menses, occurring at intervals $<24$ days. ${ }^{2}$ Dysmenorrhea is pain with menstruation. It can be primary associated with ovulatory cycles and results from myometrial contractions, in absence of demonstrable disease. Secondary dysmenorrhea refers to pain during menstruation that is associated with pelvic pathology, such as endometriosis, adenomyosis or uterine myomas. ${ }^{3}$ These are the chief complaints with which every second or third patient comes to a gynecology OPD. In addition to these, lower abdominal pain and pressure symptoms like constipation, urinary retention and frequency as well are also encountered, although infrequently in gynecology clinics. Fibroids as a cause of infertility and poor pregnancy outcomes (recurrent abortions, pre-term labour and rupture of membranes, poor placentation ) was well known since long time. Not just this, it can be easily diagnosed and cured surgically/non-surgically for better pregnancy outcomes. But adenomyosis as a cause of infertility is not yet established. However, it is being proposed that adenomyosis may lead to infertility. It may affect fertility by altered contractility of the myometrium, possible effects on implantation, and poor placentation. These factors may be responsible for poor reproduction in patients with endometriosis. ${ }^{4}$ Adenomyosis is also emerging as a cause of pre-term labor and leaking of membranes, poor placentation and IUGR. But it has been missed in the past as well as today also and it is being overlooked in work-up of AUB, RPL, infertility and 
intractable dysmenorrhea. The mechanism of these complications has been related to defective decidualization and deep placentation. ${ }^{5}$ Based on prepregnancy imaging, Juang et al. reported that adenomyosis is an important risk factor for spontaneous preterm delivery and preterm premature rupture of membranes. ${ }^{6}$ Fernando et al found that the rates of preterm birth and small for gestational age doubled in infertility patients with ovarian endometriomas who required assisted reproductive technologies (ART). ${ }^{7}$ According to Stephansson et al, endometriosis is a risk factor for preterm birth, irrespective of the type of technique of assisted reproduction performed. ${ }^{8}$

This retrospective study was carried out mainly to see the prevalence of adenomyosis in hysterectomy specimen to address the various symptoms for which patients seek gynecologic consultation. It was compared with myoma of uterus because in various studies carried out by other researchers the incidence of both the types of lesions was comparable. ${ }^{9}$ In fact in the study adenomyosis solely was responsible as the pathology in $49 \%$ of the cases and in $14 \%$ of the cases adenomyosis was found along with leiomyoma in uterine specimen. Rizvi $\mathrm{G}$ et al. observed $46.34 \%$ of hysterectomized specimen showed adenomyosis, $41.46 \%$ showed leiomyoma and $12.19 \%$ had dual pathology. ${ }^{9}$ The decreasing numbers of specimen showing fibroids in the study (37\%) can be explained as easier and earlier diagnosis by sonography and their management by either advanced drugs or by minimally accessible surgery like laparoscopic myomectomy. Neither there has been much progress in wider application of sonography to diagnose adenomyosis nor, we have very good and cheaper drugs available for non-surgical management of adenomyosis. Newer generation IUCDs (progesterone releasing), GnRH Agonists, uterine artery embolisation therapy and MRgFUS (Magnetic Resonance guided Focussed Ultrasound), all are the various treatment modalities for adenomyosis but high cost and poor availability still leaves hysterectomy as definitive treatment of choice for adenomyosis (except in infertility and RPL patients). ${ }^{10}$

\section{CONCLUSION}

Adenomyosis is a very commonly encountered pathology in gynecology patients and hence it should be kept in minds of all the gynecologists as an important differential diagnosis for patients seeking advice for AUB, dysmenorrhea, lower abdominal pain, infertility, RPL and $\mathrm{BOH}$. It is as frequent and as important as leiomyoma leading to several clinical features. Those patients coming to gynecology OPDs and ante-natal clinics with history of previous preterm deliveries and preterm pre-labour rupture of membranes are frequently sent to radiologists for ultrasound scanning of cervical length and internal-os diameter. In addition to this, it was suggested a better resolution ultrasound scanning of uterine myometrium to rule out adenomyosis. Same holds true for patients giving past obstetric history of retained placenta or manual removal of placenta. Moreover, radiologists also need to be watchful in reporting the pelvic scan of a female in reproductive age group. Adenomyosis may be one of the causes of un-explained infertility and hence a strong clinical suspicion is required in this clinical category. If diagnosed in infertility and RPL patients, prognosis and management has to be explained to the patients. As study has several advances in non-surgical management of fibroids, a satisfactory and easily available nonsurgical/drug therapy is still awaited for this prevalent uterine pathology. Adenomyosis leaves a good challenge for research in fields of gynecology, pharmacology and healthcare economics as well.

\section{ACKNOWLEDGMENTS}

Authors would like to thank Dr Geeta Pachori, H.O.D., Dept of Pathology, JLN Medical College, Ajmer for her inputs. They also thank Dr Purnima Pachori, Professor, Department of Obstetrics and Gynecology for guiding them for their support during study.

\section{Funding: No funding sources \\ Conflict of interest: None declared}

Ethical approval: The study was approved by the Institutional Ethics Committee

\section{REFERENCES}

1. Azziz R. Adenomyosis: current perspectives. Obstet Gynecol Clin North Am. 1989;16:221-35.

2. Book chapter: Abnormal Uterine Bleeding. Wolter Kluwer. Marc A. Fritz, Leon Speroff Clinical Gynecologic Endocrinology and Infertility. Eighth ed. South Asian ed: 2011; 592.

3. Menstrual disorders. Wolter Kluwer. Marc A. Fritz, Leon Speroff: Clinical Gynecologic Endocrinology and Infertility. Eighth ed. South Asian ed: 2011;579.

4. Purandare CN. Adenomyosis and Reproduction. J Obstet Gynecol India. 2006;5:387-9.

5. Brosens I, Pijnenborg R, Vercruysse L, Romero R. The "Great Obstetrical Syndromes" are associated with disorders of deep placentation. Am J Obstet Gynecology. 2011;204(3):193-201.

6. Juang CM, Chou P, Yen MS, Twu NF, Horng HC, Hsu WL. Adenomyosis and risk of preterm delivery. Brit J Obstet Gynecol. 2007;114:165-9.

7. Fernando S, Breheny S, Jaqques AM, Halliday JL, Baker G, Healy D. Preterm birth, ovarian endometriomata, and assisted reproduction technologies. Fertil Steril. 2009;91:325-30.

8. Stephansson O, Kieler H, Granath F, Falconer H. Endometriosis, assisted reproduction technology, and risk of adverse pregnancy outcome. Hum Reprod. 2009;24:2341-7.

9. Rizvi G, Pandey H, Pant H, Chufal SS, Pant P. Histopathological correlation of adenomyosis and leiomyoma in hysterectomy specimens as the cause of abnormal uterine bleeding in women in different 
age group in the Kumaon region: A retrospective study. J Mid Life Health. 2013;4:27-30.

10. Taran FA, Stewart EA, Brucker S. Adenomyosis: epidemiology, risk factors, clinical phenotype and surgical and interventional alternatives to hysterectomy. Geburtshilfe Frauenheilkd. 2013;73(9):924-31.

Cite this article as: Swati, Kumar G, Pachori P. Addressing adenomyosis: Implications beyond we actually know. Int J Reprod Contracept Obstet Gynecol 2019;8:3644-8. 